\title{
The Antiseptic Efficacy of Povidone-Iodine in Reducing Early Surgical Site Infections in Orthopaedic Implant Surgeries
}

\author{
Kelechukwu A. Okoro1', Osita Ede', Ugochukwu U. Enweani², Emmanuel C. Iyidobi', \\ Cajetan U. Nwadinigwe1, Gabriel 0. Eyichukwu' ${ }^{1}$, Udo E. Anyaehie', Francis N. Ahaotu ${ }^{1}$, \\ Richard C. Ezeh ${ }^{1}$
}

${ }^{1}$ Department of Orthopaedics and Trauma, National Orthopaedic Hospital, Enugu, Nigeria

${ }^{2}$ City Clinics, Enugu, Nigeria

Email: edeosita@yahoo.com

How to cite this paper: Okoro, K.A., Ede, O., Enweani, U.U., Iyidobi, E.C., Nwadinigwe, C.U., Eyichukwu, G.O., Anyaehie, U.E., Ahaotu, F.N. and Ezeh, R.C. (2019) The Antiseptic Efficacy of Povidone-Iodine in Reducing Early Surgical Site Infections in Orthopaedic Implant Surgeries. Journal of Biosciences and Medicines, 7, 9-18. https://doi.org/10.4236/jbm.2019.79002

Received: August 5, 2019

Accepted: August 27, 2019

Published: August 30, 2019

Copyright $\odot 2019$ by author(s) and Scientific Research Publishing Inc. This work is licensed under the Creative Commons Attribution International License (CC BY 4.0).

http://creativecommons.org/licenses/by/4.0/

\begin{abstract}
Background: Surgical site infection (SSI) is a dreaded nightmare for the Orthopaedic surgeon. Preoperative skin cleaning with antiseptics has been shown to reduce the microbial burden of the skin and results in reduced incidence of SSI. However, the ideal skin cleaning agent remains to be established. Aim: To compare the efficacy of Povidone-Iodine/Povidone-Iodine (PI-PI) combination with that of Chlorhexidine-Gluconate/Alcohol (CG-A) combination in reducing SSI in Orthopaedic surgeries. Methods: This was a prospective, randomized, double-blinded, controlled study. Subjects that met the selection criteria and gave consent were randomized into PI-PI group (test group) and the CG-A group (control group). Both the patients and the assessors for SSI were blinded to the group a participant belongs. Blocking was done on the type of surgery to cancel the confounding effect of surgery type on SSI. Standard perioperative protocols were applied to both groups. Assessment for features of SSI was done on the $3^{\text {rd }}$ day, $7^{\text {th }}$ day, $14^{\text {th }}$ day, $6^{\text {th }}$ week and $12^{\text {th }}$-week postoperative period. The diagnosis of SSI was made based on the Centers for Disease and Control (CDC) guidelines. Results: We recruited 124 patients for this study, 62 males and 54 females. The mean age of the subjects was 37.5 years ( $\mathrm{SD}=14.7$ years). Sixty-two subjects were randomized into each group. There was no significant difference in the distribution of the genders in the study arms. Other possible confounders such as duration of hospital stay, use of drains, the surgeon involved and age were evenly distributed in the two groups. Eight patients did not complete the study. The overall incidence of SSI in the study was $2.6 \%$. Subjects in the control group had an SSI of $3.4 \%$ while those in the PI-PI group had a rate of $1.8 \%$; however, this was not significant, $\mathrm{p}=0.579$. Conclusion: Both CG-A
\end{abstract}


and PI-PI combinations are equally efficacious as preoperative skin antiseptic in Orthopaedic implant surgeries.

\section{Keywords}

SSI, Orthopaedic Implant Surgeries, Chlorhexidine Gluconate-Alcohol, Povidone Iodine

\section{Introduction}

Surgical site infection (SSI) is infection occurring within the surgical wounds, or within organs entered or manipulated during the surgical procedure, within 30 days after surgery or within one year if an implant is used and left in-situ [1]. The Centers for Disease and Control (CDC) has classified SSI into incisional and organ/space infection, with the former further subdivided into superficial and deep [1]. Incisional SSI is the most common type with most infection occurring within 30 days, peaking within the $5^{\text {th }}$ to $10^{\text {th }}$ postoperative period. The worldwide incidence of SSI is between $2.6 \%$ and $41.9 \%$ [2]. In Nigeria, the rate of SSI in Orthopaedic surgeries ranges from $4.6 \%$ to $16.7 \%$ [3]-[9].

Surgical site infections increase patients' morbidity and mortality. It increases the overall cost of the patient's care. These costs are incurred through increased lengths of hospital stay, prices of materials for wound care, pharmacy costs for antibiotics, increased outpatient and emergency room visits, diagnostic laboratory studies, reoperation rate, and physician expenses. Mortality can result from septicaemia and multiple organ failure or indirect consequence of prolonged admission, such as pulmonary embolism. The annual cost of treating SSI in the United States is $\$ 10$ billion [10]. In Australia, the conservative estimate of annual direct healthcare costs of treating superficial wound complications following prosthetic joint replacement is $\$ 34$ million [11].

Preoperative skin antisepsis has been shown to reduce the epidermal bacterial load [12]. Their routine use in surgery has been recommended by professional and public health organizations including the Royal College of Surgeons of England, National Institute for Health and Care Excellence (NICE) and the United States Centre for Disease Control and Prevention (CDC) [13] [14] [15]. The three main preoperative skin antiseptics are iodine or iodophor, alcohol, and aqueous or alcoholic chlorhexidine gluconate [15]; however, the question of which is superior is still unresolved, with studies giving conflicting results. At our institution, we routinely use a combination of $4 \%$ Chlorhexidine Gluconate and $70 \%$ Isopropyl alcohol to prepare our skin site. We intend to compare the antiseptic efficacy of a combination of $7.5 \%$ Povidone Iodine and $10 \%$ Povidone Iodine to our routine antiseptic combination.

\section{Subjects and Methods}

This work was conducted at the National Orthopaedic Hospital, Enugu in 
South-East Nigeria, from November 2017 to October 2018. It is a tertiary hospital and regional centre for Orthopaedics and Trauma. Ethical approval was obtained from the hospital's Research, Ethics and Training Committee under IRB/HEC number S.313/IV and protocol number 942. Written informed consent was obtained from the patients recruited for the study. The minimum sample size for the study was determined using the formula to compare two independent proportions as shown [16]:

$$
n=2(Z \alpha+Z 1-\beta) 2 P Q / E^{2}
$$

where $P=(p 1+p 2) / 2$ and $Q=(1-P), E=p 1-p 2, n=$ minimum sample size for each group, $Z \alpha=$ standard normal deviate, at a confidence level of $95 \%=$ $1.96, Z \beta=0.842$ at $80 \%$ power, $p 1=$ surgical infection rate with chlorhexidine, $2 \%$ [17], $p 2=$ surgical infection rate with povidone-iodine, $17.9 \%$ [18], $n=$ $2(1.96+0.84)^{2}(0.1)(0.9) /(0.16)^{2}, n=55.125$. We anticipate an attrition rate of $10 \%$; hence, the sample size was adjusted by a factor, $q$, which is given by $q=1 /(1-f)$ where $f$ is the estimated non-response rate. Hence the minimum sample size for the study was $1.11(55.125)=61.189$, which is rounded up to 62 . Therefore, each study arm had 62 participants each.

The inclusion criteria were all patients 18 years and above undergoing orthopaedic implant surgeries at the main theatre of the hospital and who gave informed consent to the study. The exclusion criteria were open wounds at the site of surgery, clinical/microbiological evidence of infection at/adjacent to the surgical site or at distant site, pathologic fractures, morbid obesity (BMI > 35 $\mathrm{kg} / \mathrm{m}^{2}$ ), cigarette smoking within two weeks of surgery, peripheral vascular disease, ongoing systemic sepsis, immunocompromised conditions e.g. DM, HIV, chronic steroid use, known allergy to chlorhexidine, iodophors or alcohol.

Blocking was first done on the type of surgery. The surgery was divided into arthroplasty, spine surgery, trauma and others. From within each block, randomization was done to either the control group (CG-A) or the test group (PI-PI). We used the random number generator application downloaded from Google play store. If an even number is generated, the subject is allocated to the control group. If an odd number is generated, the participant is allocated to the test group. Whenever a subject from a block is allocated to a treatment group, the next subject from the same block is automatically allocated to the other group.

The operative area, in the control group, was scrubbed with $4 \%$ Chlorhexidine gluconate in water for 5 minutes, dried and then painted with 70\% isopropyl alcohol, which is allowed to air dry. In the test group, scrubbing was with $7.5 \%$ Povidone-iodine for 5 minutes, and then 10\% Povidone-iodine was then used to paint the skin. The hospital's pharmacy department provided both antiseptics. Prophylactic antibiotic (Intravenous Ceftriaxone $1 \mathrm{~g}$ ) was given at induction of anaesthesia in both groups and tourniquet, where applicable, was applied ten minutes after antibiotics were given.

Postoperatively, two further courses of Ceftriaxone were given, consistent 
with the hospital's protocol. Drains, if used, were removed when the wound drain is less than $50 \mathrm{mls}$ the previous day. Two designate senior registrars who were blinded to the treatment group of the subject, inspected the wounds for signs of SSI on the 3rd, 7th and 14th postoperative days, and on the 6th and 12th postoperative week. However, if there is a suspicion of SSI, the wound was examined immediately, and swabs were taken for microscopy, culture and sensitivity analysis and appropriate protocol of wound care commenced. Other postoperative protocols, including analgesia, anticoagulation and physiotherapy were identical in both groups. Skin reactions were also monitored in both groups.

The diagnosis of SSI was made based on the CDC guidelines [1]. The two senior registrars had to agree on a clinical diagnosis of SSI, where a disagreement occurred, a culture result was used to settle it. Results were presented as means and standard deviations for continuous variables and as frequencies for categorical variables. Statistical analysis was done with IBM SPSS version 20. A Chi-square analysis was used to test for significant differences in the rate of SSI in the two groups. It was also used to test for any difference in the incidence of cofounding variables between the two groups. All the tests were 2-tailed, and a p-value less than 0.05 was considered significant.

\section{Results}

There were 62 males (53.4\%) and 54 females (46.6\%). Table 1 shows the sex distribution across the study groups with a male: female ratio of 1.14:1. Eight patients did not complete the study, three from CG-A arm (two patients were lost to follow up, one had a breach of asepsis intraoperatively) and five from PI-PI division (three patients were lost to follow up, one had a violation of asepsis intraoperatively, and one died before completion of the 90-day follow up). Thus, 116 patients completed the study, 59 in the CG-A arm and 57 in in the PI-PI group.

The age of the patients ranged from 18 - 68 years. There was no significant difference in the mean age of the study groups, $p=0.16$. The preoperative and postoperative duration of admission in the two groups does not differ significantly. Table 2 details the age distribution and period of hospitalization in the two groups.

Fracture fixation accounted for the majority of the surgeries. Figure 1 illustrates the range of operations done in the study.

A Consultant did the surgery in $70.2 \%$ of cases in the Povidone-iodine group and $76.3 \%$ in the Chlorhexidine group ( $\mathrm{p}=0.458)$. Wound drain was used in 36 patients $(61 \%)$ of the CG-A arm and 35 patients $(61 \%)$ of the PI-PI arm ( $\mathrm{p}=$ $0.575)$.

The overall incidence of SSI in this study was $2.6 \%$ (three cases only), see Table 3. All were superficial Incisional Primary Infections. There were no deep SSIs or Organ/Space SSIs.SSI occurred in 2 cases (3.4\%) of the CG-A arm and only one case $(1.8 \%)$ of the PI-PI arm. However, this difference was not statistically significant, $\mathrm{p}=0.579$. Escherichia coli was isolated in two instances of SSI while 
mixed isolates of Escherichia coli, Klebsiella spp., and Staphylococcus aureus were found in the third case of SSI. Adverse skin reaction (bullae) occurred in 6 patients $(10.5 \%)$ in the Povidone Iodine-Povidone Iodine group, while none occurred in the Chlorhexidine-Alcohol group. This difference was statistically significant $(\mathrm{p}=0.013)$.

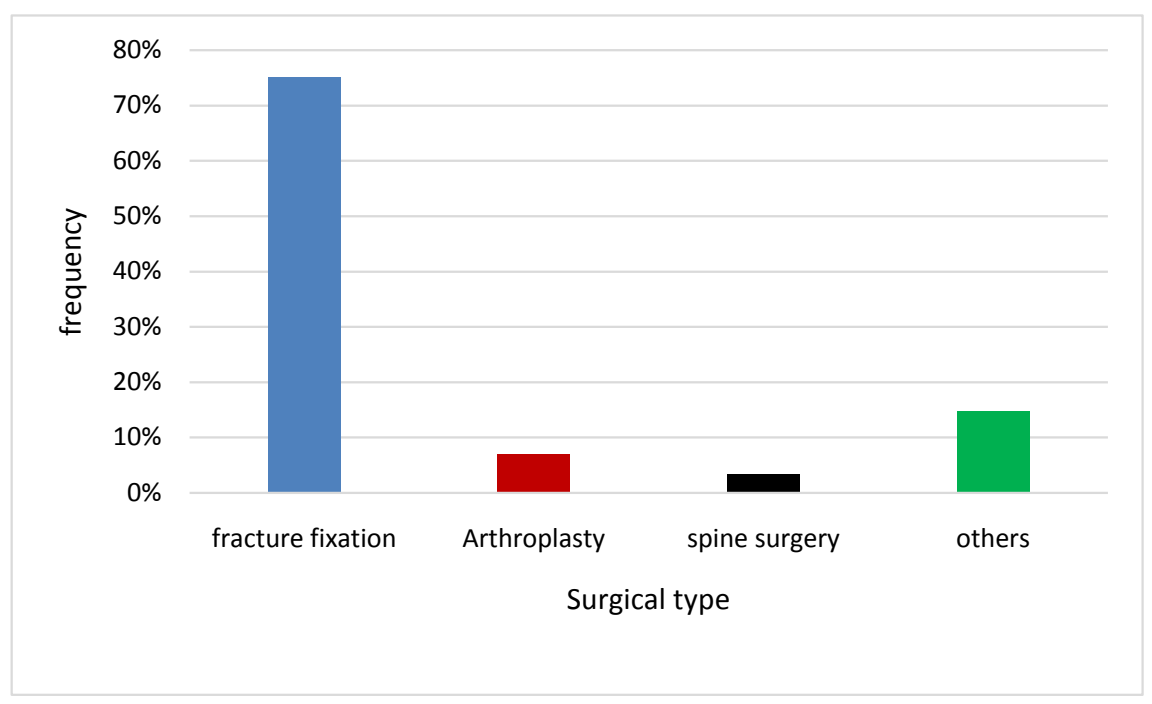

Figure 1. Surgeries done for the subjects. Others include corrective osteotomies, non-unions and pinning for SUFE. Spine surgery is posterior lumbar decompression with pedicle screw fixation.

Table 1. Sex distribution across the study group $(n=116)$.

\begin{tabular}{cccc}
\hline Study group & Male & Female & Total \\
\hline Chlorhexidine-Alcohol & 31 & 28 & 59 \\
Povidone-iodine-Povidone-iodine & 31 & 26 & 57 \\
Total & 62 & 54 & 116 \\
\hline
\end{tabular}

The table shows that gender was homogeneously distributed across the study groups.

Table 2. The mean age and mean duration of admission.

\begin{tabular}{cccc}
\hline Variable & Group & Mean & Stand. Deviation \\
\hline Age (years) & CG-A & 37.5 & 14.7 \\
& PI-PI & 41.8 & 15.1 \\
$\begin{array}{c}\text { Duration of preoperative } \\
\text { admission (days) }\end{array}$ & CG-A & 5.3 & 5.0 \\
$\begin{array}{c}\text { Duration of postoperative } \\
\text { admission (days) }\end{array}$ & PI-PI & 6.2 & 5.1 \\
& CG-A & 8.6 & 3.7 \\
& PI-PI & 9.7 & 4.6 \\
\hline
\end{tabular}

CG-A = Chlorhexidine Gluconate-Alcohol. PI-PI = Povidone Iodine-Povidone Iodine. 
Table 3. The distribution of wound infection between the two groups.

\begin{tabular}{ccc}
\hline Agent & Frequency & Percent \\
\hline CG-A & 2 & 3.4 \\
PI-PI & 1 & 1.8 \\
\hline
\end{tabular}

Adverse skin reactions occurred in six patients (10.5\%) of the PI-PI group and were not seen in the CG-A group. There were four cases of rashes around the wound, one blister and one erythema associated with pruritus. All resolved by the third week following surgery.

\section{Discussion}

This work has shown that there is no difference in the efficacy of both Chlorhexidine Gluconate-Alcohol combination and Iodophor combination in reducing early SSI. We limited our selection to clean Orthopaedic surgeries were an implant is used. We also blocked on type of surgery, which we believe can influence the incidence of SSI. From the results, it can also be seen that other possible confounding variables were evenly distributed in the two groups. The reason is to minimize the influence of these external factors on the observed response.

Aurobinda et al. [18] compared the efficacy of 4\% CG-4\% CG and 7.5\% PI-10\% PI in preventing SSI in 420 elective orthopaedic procedures. It was a multicentre prospective study with similar inclusion and exclusion criteria that divided the cases into two equal study groups; the patients were followed up for three weeks. The SSI rate in the PI-PI group was $12.3 \%$, which is higher than the $1.8 \%$ reported in this study. The higher rate may be attributed to the multicentred nature of the study with variations in perioperative protocols. Also, the level of expertise of the surgeons was not stated in the research.

Another close study conducted by Paocharoen et al. [17] on a subset of 183 participants who underwent clean elective foot and ankle surgeries, and followed up for one month, reported SSI rates of $2.1 \%$ and $5.7 \%$ in the $4 \%$ CG/70\% Isopropyl alcohol and 7.5\% PI/10\% PI groups respectively. These SSI rates are closer to those obtained in this study. Similarly, there was no significant difference between these rates, $95 \% \mathrm{CI}$ [0.55 to 13.86].

In another related study, Saltzman et al. [19] conducted a three-arm trial that randomized 150 participants undergoing clean shoulder surgeries (implants inclusive) into three groups: $7.5 \%$ PI with $10 \%$ PI, $2 \%$ CG with $70 \%$ isopropyl alcohol and $0.7 \%$ iodophor with $74 \%$ isopropyl alcohol. There were no reported SSI events in the postoperative surgical wounds randomized to either 7.5\% PI $/ 10 \%$ PI or $2 \%$ CG/70\% alcohol groups over a 10 -month follow up period.

Extending to other non-orthopaedic cases, the rate of SSI in the CG-A study arm is similar to the $2.2 \%$ SSI rate reported by Swenson et al. for clean general surgery cases. [20] Whereas 4\% CG was used in this study, they used 2\% CG. Darouiche et al. [21] reported an SSI rate of 9.5\% even though 2\% CG was used 
for skin preparation and the subjects recruited underwent clean contaminated abdominal and thoracic surgeries.

Conversely, Swenson et al. [20] documented an SSI rate of $0.84 \%$ when $10 \%$ PI was used as skin preparation in clean general surgery cases. This is similar to the SSI rate of $1.8 \%$ noted in the PI-PI arm of this study. Darouiche et al. [21] reported a $16.1 \%$ rate of SSI following $10 \%$ PI skin preparation for clean-contaminated surgeries while Srinivas et al. [22] recorded an SSI rate of $17.9 \%$. In the latter study, however, $0.5 \%$ PI was used for skin preparation, and the patients had elective clean-contaminated upper abdominal surgeries. The inclusion of only clean surgeries as opposed to clean contaminated may have been responsible for the lower SSI rates found in this study.

The overall incidence of SSI in this study was $2.6 \%$, which is higher than the accepted standard of less than 1\% [23]. This is similar to the SSI rate of 3.0\% reported by Ogugua et al. [24] in the same institution. This is lower than that obtained from other local studies by Onche et al. [4], Madu [7], and Ngim et al. [25] who documented SSI rates of 7.5\%, 9.3\%, and 9.38\% respectively following orthopaedic implant surgeries. In another third world country, Amaradeep et al. [20] and Sunnet et al. [26] recorded higher rates of $4.44 \%$ and $6.89 \%$ respectively for elective orthopaedic implant surgeries. The difference in study methodologies, surgical setups, facilities and endpoints may have accounted for the variance in the SSI rates documented in these studies.

Adverse skin reactions occurred in 6 patients (10.5\%) in the PI-PI study arm while none were observed in the CG-Alcohol study arm. These were judged to be related to study solution, and they manifested as erythema and bullae around the surgical wound. There were no cases of fire, chemical or diathermy skin burns in the theatre. Daroiuche et al. [21] reported similar events (pruritus, erythema, or both around the surgical wound) in the $0.7 \%$ of patients who had skin preparation with $10 \%$ Povidone Iodine. However, there were no such adverse events reported by Srinivas et al. [22], who used 5\% Povidone-iodine as the study skin preparation solution. This may have been because a lower concentration of PI was used, although this requires further validation. In the same study, there were no such events following CG-Alcohol application even though the concentration of CG was $0.5 \%$.

\section{Conclusion}

There is no significant difference in the risk of SSI with either Chlorhexidine Gluconate-Alcohol combination or Iodophor combination antiseptic in clean orthopaedic implant surgery. Skin preparation for clean orthopaedic implant surgeries should be done with either of the two while factoring the risk of adverse skin reaction reported with Povidone-Iodine solution.

\section{Funding}

The hospital management provided a financial grant for this work. 


\section{Acknowledgements}

We wish to thank all the Consultants whose patients were used for this study.

\section{Conflicts of Interest}

The authors declare no conflicts of interest regarding the publication of this paper.

\section{References}

[1] Horan, T.C., Gaynes, R.P., Martone, W.J., Jarvis, W.R. and Emori, T.G. (1992) CDC Definitions of Nosocomial Surgical Site Infections, 1992: A Modification of CDC Definitions of Surgical Wound Infections. Infection Control \& Hospital Epidemiology, 13, 606-608. https://doi.org/10.1017/S0195941700015241

[2] Davenport, M. and Doig, C.M. (1993) Wound Infection in Pediatric Surgery: A Study in 1,094 Neonates. Journal of Pediatric Surgery, 28, 26-30. https://doi.org/10.1016/S0022-3468(05)80348-3

[3] Montefiore, D., Alausa, K.O., Cooke, A.R., Familusi, J.B., Ojo, O.A., Oyediran, A.B.O.O., et al. (1979) Epidemiological Surveillance of Hospital-Acquired Wound Infections. Nigerian Medical Journal, 9, 289-293.

[4] Onche, I. and Adedeji, O. (2004) Microbiology of Post-Operative Wound Infection in Implant Surgery. Nigerian Journal of Surgical Research, 6, 37-40. https://doi.org/10.4314/njsr.v6i1-2.54787

[5] Enweani, U.N. (1991) Surgical Wound Sepsis in Clean Orthopaedic Procedures: Bacteriology and Sensitivity in a Regional Specialist Centre. Orient Journal of Medicine, 3, 3-6.

[6] Nwankwo, O.E., Eze, B., Onabuwale, B.O. and Osisioma, E.C.O. (1990) Infection Rate Using Austin Moore Prosthesis for Partial Replacement Arthroplasty of the Hip in an Ordinary Operating Theatre: 11-Year Experience at the National Orthopaedic Hospital Enugu. Orient Journal of Medicine, 2, 66-69.

[7] Madu, K.A. (2008) Post-Operative Wound Infection in Implant Surgery at the National Orthopaedic Hospital Enugu. A Dissertation Submitted to the National Postgraduate Medical College of Nigeria, Lagos.

[8] Ikeanyi, U.O.E. (2007) Postoperative Wound Infections in Clean Cases-Incidence, Risk Factors and Bacteriology at the National Orthopaedic Hospital Igbobi, Lagos. A Dissertation Submitted to the National Postgraduate Medical College of Nigeria, Lagos.

[9] Mbamali, E.I. (1981) Internal Fixation of Femoral Shaft Fractures at the Ahmadu Bello University Teaching Hospital, Zaria. Nigerian Medical Practitioner, 2, 81-85.

[10] Scott, R.D. (2009) The Direct Medical Costs of Healthcare-Associated Infections in U.S. Hospitals and the Benefits of Prevention. Centers for Disease Control and Prevention, Atlanta.

[11] Kirkland, K.B., Briggs, J.P., Trivette, S.L., Wilkinson, W.E. and Sexton, D.J. (1999) The Impact of Surgical-Site Infections in the 1990s: Attributable Mortality, Excess Length of Hospitalization, and Extra Costs. Infection Control \& Hospital Epidemiology, 20, 725-730. https://doi.org/10.1086/501572

[12] Ingi Lee, M.M., Rajender, K., Agarwal, M.M., et al. (2010) Systematic Review and Cost Analysis Comparing the Use of Chlorhexidine with Use of Iodine for Preoperative Skin Antisepsis to Prevent Surgical Site Infection. Infection Control \& Hospit 
al Epidemiology, 31, 1219-1229. https://doi.org/10.1086/657134

[13] Mangram, A.J., Horan, T.C., Pearson, M.L., et al. (1999) Guideline for Prevention of Surgical Site Infection, 1999. Hospital Infection Control Practices Advisory Committee. Infection Control \& Hospital Epidemiology, 20, 250-278.

https://doi.org/10.1086/501620

[14] Leaper, D.J., Orr, C., Maung, Z. and White, A. (2001) Inflammation and Infection. Step 2000 Module II. Royal College of Surgeons of England, Blackwell Science, London.

[15] Edwards, P., Lipp, A. and Holmes, A. (2009) Preoperative Skin Antiseptics for Preventing Surgical Wound Infections after Clean Surgery. Cochrane Database of Systematic Reviews, No. 3, CD003949.

[16] Habib, A., Johargy, A., Mahmood, K. and Humma, H. (2014) Design and Determination of the Sample Size in Medical Research. IOSR Journal of Dental and Medical Sciences, 13, 21-31. https://doi.org/10.9790/0853-13562131

[17] Paocharoen, V., Mingmalairak, C. and Apisarnthanarak, A. (2009) Comparison of Surgical Wound Infection after Preoperative Skin Preparation with 4\% Chlorhexidine and Povidone Iodine: A Prospective Randomized Trial. Journal of the Medical Association of Thailand, 92, 898-902.

[18] Aurobinda, D., Saswat, S. and Pragyan, D. (2017) A Comparison Study of Preoperative Skin Preparation Using Chlorhexidine vs Povidone Iodine in Cases of Elective Orthopaedic Surgery. International Journal of Orthopaedics Sciences, 3, 12-15. https://doi.org/10.22271/ortho.2017.v3.i2a.03

[19] Saltzman, M.D., Nuber, G.W., Gryzlo, S.M., Marecek, G.S. and Koh, J.L. (2009) Efficacy of Surgical Preparation Solutions in Shoulder Surgery. Journal of Bone and Joint Surgery, 91, 1949-1953. https://doi.org/10.2106/JBJS.H.00768

[20] Swenson, B.R., Hedrick, T.L., Metzger, R., Bonatti, H., Pruett, T.L. and Sawyer, R.G. (2009) Effects of Preoperative Skin Preparation on Postoperative Wound Infection Rates: A Prospective Study of 3 Skin Preparation Protocols. Infection Control \& Hospital Epidemiology, 30, 964-971. https://doi.org/10.1086/605926

[21] Darouiche, R.O., Wall, M.J.J., Itani, K.M., Otterson, M.F., Webb, A.L., Carrick, M.M., et al. (2010) Chlorhexidine-Alcohol versus Povidone-Iodine for Surgical-Site Antisepsis. The New England Journal of Medicine, 362, 18-26. https://doi.org/10.1056/NEJMoa0810988

[22] Srinivas, A., Kaman, L., Raj, P., Gautam, V., Dahiya, D., Singh, G., Singh, R. and Medhi, B. (2015) Comparison of the Efficacy of Chlorhexidine Gluconate versus Povidone Iodine as a Preoperative Skin Preparation for the Prevention of Surgical Site Infections in Clean-Contaminated Upper Abdominal Surgeries. Surgery Today, 45, 1378-1384. https://doi.org/10.1007/s00595-014-1078-y

[23] Amaradeep, G., Shiva, P.S.S. and Manjappa, C.N. (2017) Surgical Site Infections in Orthopaedic Implant Surgery and Its Risk Factors: A Prospective Study in a Teaching Hospital. International Journal of Orthopaedics Sciences, 3, 169-172. https://doi.org/10.22271/ortho.2017.v3.i3c.28

[24] Ogugua, J.H. (2014) Surgical Site Infection in Implant Surgery. Dissertation Submitted to the National Post-Graduate Medical College of Nigeria, Lagos, 35-62.

[25] Ngim, N.E., Etokidem, A.J., Ikpeme, I.A. and Udosen, A.M. (2013) Surgical Site Infection in Clean Orthopaedic Operations: Experience from the Third World. Asian Journal of Medical and Clinical Sciences, 2, 30-32.

[26] Suneet, T., Abhishek, P., Santosh, K.M. and Mayank, V. (2015) Incidence and Risk 
Factors for Early Surgical Site Infection in Elective Orthopaedic Implant Surgeries: A Prospective Study. Journal of Evolution of Medical and Dental Sciences, 4, 2505-2511. 